See discussions, stats, and author profiles for this publication at: https://www.researchgate.net/publication/235755932

\title{
Weight loss and clinical characteristics of young adults patients seeking treatment at medical centers: Data from the QUOVADIS Study
}

Article in Eating and weight disorders: EWD · December 2012

DOI: 10.1007/BF03325141 · Source: PubMed

\section{CITATIONS}

2

6 authors, including:

Simona Calugi

Villa Garda Hospital

174 PUBLICATIONS 2,703 CITATIONS

SEE PROFILE

Angelo Compare

University of Bergamo

170 PUBLICATIONS 1,374 CITATIONS

SEE PROFILE
READS

61

Riccardo Dalle Grave

Villa Garda Hospital, Italy

246 PUBLICATIONS 3,648 CITATIONS

SEE PROFILE

Maria Letizia Petroni

82 PUBLICATIONS 2,274 CITATIONS

SEE PROFILE

Some of the authors of this publication are also working on these related projects:

Project The role of external maintaining factors in the outcome of outpatient CBT-E View project

Project Metabolism in liver transplantation View project 


\title{
$\underset{\text { REPORT }}{\text { BRIEF }}$ Weight loss and clinical characteristics of young adults patients seeking treatment at medical centers: Data from the QUOVADIS Study
}

\author{
S. Calugi ${ }^{1}$, R. Dalle Grave ${ }^{1}$, A. Compare ${ }^{2}$, E. Dall'Aglio ${ }^{3}$, M.L. Petroni, \\ and G. Marchesini ${ }^{5}$, for the QUOVADIS Study Group
}

${ }^{1}$ Department of Eating and Weight Disorder, Villa Garda Hospital, Garda (VR), ${ }^{2}$ Department of Human Sciences, University of Bergamo, Bergamo, ${ }^{3}$ Unit of Clinical Dietetics and Diabetes, Maggiore Hospital, Parma, ${ }^{4}$ Nutrition and Rehabilitation Unit, IRCCS Italian Auxologic Institute, Piancavallo (VB), ${ }^{5}$ Unit of Metabolic Diseases and Clinical Dietetics, "Alma Mater Studiorum” University, Bologna, Italy

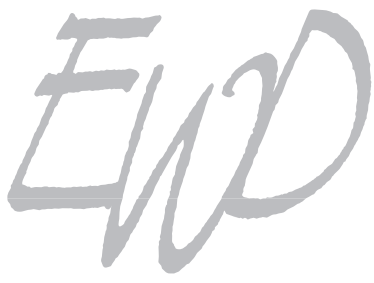

ABSTRACT. OBJECTIVE: To compare clinical characteristics, attrition, weight loss, and psychological changes of obese young adults and obese adults seeking treatment. MATERIALS AND METHODS: 1530 individuals seeking treatment in 18 Italian medical centers were evaluated. 382 cases (25\%) were classified as young adults (age $\leq 35$ years), 1148 (75\%) as adults (>35 years). Psychological distress, binge eating, body uneasiness, and attitude towards eating were evaluated, at baseline and after a 12-month weight-loss program, together with BMI changes. Weight-loss expectations and primary motivation for seeking treatment were also recorded. RESULTS: At baseline, young adults reported significantly higher BMI at age 20, weight loss expectations and body uneasiness scores than adults. A significantly higher percentage of young adults also reported improving appearance as primary reason for seeking treatment. The attrition rate was significantly larger in young adults. Among completers, the mean percent weight loss at 12 months and improvement of psychosocial variables were significantly higher in young adults than in adults. By intention to treat, BMI changes were no longer significant between groups. DISCUSSION: Obese young adults lose more weight and considerably improve psychological distress, but show a higher attrition rate after 12 months of continuous care in a real world medical setting.

(Eat. Weight Disord. 17: e314-e319, 2012). ๑2012, Editrice Kurtis

\section{INTRODUCTION}

The prevalence of overweight and obesity in the young adults of western countries is epidemic, reaching about $50 \%$ in the United States (1). Early adulthood is a period of life at high risk of weight gain (1-4); body weight increases on average by 1-2 lb per year during this period, in particular in individuals who are already overweight $(3,4)$. These data are alarming, particularly because weight gain and obesity in young adults are associated with an increased risk of severe obesity (5), cardiovascular diseases $(3,6)$, and disability problems (e.g. knee osteoarthritis) later in life (7). There is an urgent need for weight gain prevention programs in the community (8) and weight loss effective treatments for young adults who are already overweight.

A few randomized weight gain prevention trials involving young adults produced encouraging short-term results and report- ed very few or no changes in body weight in the intervention groups and weight gain in the control arms (9-11). Unfortunately, the long-term results of two studies were discouraging, showing a significant weight gain over the 3-year study period $(8,12)$.

Very few data are available on the treatment of obese young adults, an intermediate-risk age group that seems to have distinctive clinical characteristics from other obese subjects. Most studies had small sample sizes, short-term evaluation and lack of follow-up (13). Very few young adults (defined from 18 to 35 years of age) were retrieved in three NIH-funded adult behavioral weight loss trials from two clinical centers (14). They represented only $7 \%$ (21 subjects) of the sample, attended fewer sessions and had a lower retention rate than adults. In addition, young adults lost a lower amount of weight than adults, but the difference was no longer significant in com- 
pleters. The authors concluded that it is urgent to improve recruitment and retention efforts in high-risk obese young adults. No study evaluated the treatment outcome of these patients in real world settings and no data are available on their psychosocial characteristics.

Aims of this study were to assess clinical characteristics, treatment adherence and outcome of obese young adults participating in an observational Italian study with a 12-month follow-up (15). In our sample, we classified patients from 18 to 35 years of age as "young adults", while patents over 35 were classified as "adults", according to Erikson (16).

\section{MATERIALS AND METHODS}

QUOVADIS Study Planning and protocol The QUOVADIS (QUality of life in Obesity: eVAluation and DIsease Surveillance) study planning and protocol have been described in details in a previous paper (15). QUOVADIS is a purely observational study on quality of life, psychological distress, and eating behavior in obese patients seeking treatment at 25 obesity medical centers accredited by the Italian Health Service and treated according to their specific protocols. All the centers adopted an eclectic approach including different forms of dieting and physical exercise, combined in some cases with basic behaviormodifying strategies (e.g., food diary), drugs and bariatric surgery (less than $2 \%$ of patients). Whilst the theory on which the treatment was based varied between centers, all adopted an initial intensive treatment period (3-6 months), followed by a less intensive continuous care (a follow-up control every 2-4 months) with periodic controls for an indefinite period of time (17).

All individuals with obesity (BMI $\left.\geq 30 \mathrm{~kg} / \mathrm{m}^{2}\right)$, consecutively seeking treatment, including those with binge eating, were eligible for the study, provided they were not on active treatment at the time of enrolment, were in the age range between 25 and 65 and agreed to complete a set of self-administered questionnaires. To ensure that subjects were truly consecutive, the medical record number of the patients was monitored across sites.

A systematic medical and psychosocial evaluation of participants was planned at baseline, approximately one week before the beginning of treatment, and after 12 months. Seven centers refused to collect the 12-month follow-up data because of limited human resources and longitudinal data were thus available only for 18 centers. The present analysis is based on the longitudinal data of subjects on continuous care at 12 months. For the purposes of the pre- sent report, six cases who underwent bariatric surgery were excluded from analysis.

The protocol was approved by the ethical committees of each center, after approval by the ethical committee of the coordinating center (Azienda Ospedaliera di Bologna, Policlinico S. Orsola-Malpighi). All participants gave written informed consent for participation.

\section{Measures}

Case Report Form. The Case Report Form was filled in by physicians at the time of enrollment by directly interviewing patients. It included demographic data and a detailed diet history, with specific information on the number and the results of previous attempts to lose weight, age at first dieting, expected one-year weight loss, maximum acceptable weight and dream weight. Expected one-year loss was defined as "the weight loss that patients were expecting to lose with treatment in the following 12 months". Maximum acceptable weight was defined as "the heaviest body weight that patients could accept and tolerate," whereas dream weight was defined as "the body weight that they were dreaming to achieve, however unrealistic it was." The form also included a question on the reasons for seeking treatment. For this specific purpose, patients were asked to choose their primary motivation among three possible answers: 1) improving appearance, 2) improving future health, and 3) improving present health.

Psychosocial Measures. At baseline and at follow-up, the participants completed a battery of questionnaires measuring psychological distress and binge eating.

The Symptom CheckList-90R (SCL) (18) was used to identify psychological distress. For each item, patients marked how much that problem had distressed them during the previous week, with responses ranging from 0 (not at all) to 4 (extremely). The 90 items of the test were used to compute the general symptom index (GSI), which is an indicator of the overall psychological distress (18). The various subscales of SCL were not considered for the purposes of the present study. The internal consistency coefficient alphas for the nine symptom dimensions of SCL ranged from 0.77 to 0.90 (18).

The Three-Factor Eating Questionnaire (19), later renamed Eating Inventory (EI) (20) was used to evaluate attitudes toward eating. The EI has three scales: 1) dietary (cognitive) restraint, 2) disinhibition, and 3) hunger. Dietary restraint measures the cognitive ability to restrain food intake. Disinhibition refers to one's tendency to lose control over eating. Hunger refers to the perception of hunger/satiety. The EI has good internal consistency $(<0.80)(19)$ and the reliabil- 
ity and validity of its scales have been established in many studies (21).

The Body Uneasiness Test (BUT) was used to evaluate body uneasiness (22). The term 'body uneasiness' was used to describe not only body dissatisfaction but also body-associated emotions, such as anxiety, alarm, trepidation, worry, mistrust, misgiving, doubt, suspicion and embarrassment. The BUT consists of two parts: 1) BUTA comprises 34 items with a score ranging from 0 (never) to 5 (always). The scores of the 34 items are used to compute the global severity index (GSI); 2) BUT-B has 37 items that refer to specific worries about particular body parts or functions. For the aim of the present study we only used Part A, since BUT-B also evaluates worries on specific body parts (e.g., mouth, moustaches, and skin) that are not modifiable with weight loss. BUT was validated in patients with obesity and showed good internal consistency (Cronbach's alpha coefficients greater than 0.7) (23).

The Binge Eating Scale (BES) was used to measure the severity of binge eating (24). examines both behavioral signs (eating large amounts of food) and feeling or cognition during a binge episode (loss of control, guilt, fear of being unable to stop eating) in 16 items.

Weight and Height. Weight was measured on a medical-balance and height by a stadiometer in patients with underwear and without shoes. Weight change was examined from baseline to 12 months.

\section{Statistical analyses}

Chi-square test or t-test were used to compare "young adults" and "adults" (16) on demographic and clinical variables. Repeated-measure analysis of variance was carried out on the completers in order to analyze the time-related changes from baseline to 12-month follow-up in BMI and in psychosocial variables between groups. Subsequently, using an intention-totreat approach and the baseline carried-forward method for missing data, we repeated the analysis of variance on the whole sample.

Data are reported as mean \pm standard deviation (SD) or percentage. All analyses were performed using SPSS for Windows, Version 15.0.

\section{RESULTS}

\section{Baseline characteristics}

The characteristics of the whole QUOVADIS sample at baseline were described in detail in previous reports $(15,17)$.

TABLE 1

Baseline data of patients included in the analysis (means \pm SD or percent).

\begin{tabular}{|c|c|c|c|c|}
\hline$8 0 \longdiv { 2 }$ & $\begin{array}{l}\text { Young adults } \\
\qquad(\mathrm{N}=382)\end{array}$ & $\begin{array}{c}\text { Adults } \\
\text { (N=1148) }\end{array}$ & $\begin{array}{c}\text { T-test or Chi-Square } \\
\text { test }\end{array}$ & $p$ \\
\hline Gender, F, N (\%) & $301(78.8)$ & $893(74.8)$ & 0.17 & 0.680 \\
\hline BMI $\left(\mathrm{kg} / \mathrm{m}^{2}\right)$ & $38.3 \pm 6.5$ & $37.8 \pm 6.2$ & 1.47 & 0.141 \\
\hline \multicolumn{5}{|l|}{ Historical Variables } \\
\hline BMl at age $20\left(\mathrm{~kg} / \mathrm{m}^{2}\right)$ & $28.7 \pm 6.0$ & $24.8 \pm 4.4$ & 11.88 & $<0.001$ \\
\hline Maximum BMI $\left(\mathrm{kg} / \mathrm{m}^{2}\right)$ & $39.6 \pm 6.9$ & $38.8 \pm 6.6$ & 1.93 & 0.054 \\
\hline Maximum weight loss (\%) & $19.1 \pm 9.6$ & $18.8 \pm 9.3$ & 0.35 & 0.723 \\
\hline \multicolumn{5}{|l|}{ Weight Loss Expectations } \\
\hline Maximum Acceptable BMI $\left(\mathrm{kg} / \mathrm{m}^{2}\right)$ & $27.9 \pm 4.0$ & $29.4 \pm 4.1$ & -6.50 & $<0.001$ \\
\hline Dream BMI $\left(\mathrm{kg} / \mathrm{m}^{2}\right)$ & $24.6 \pm 2.9$ & $26.5 \pm 3.4$ & -9.43 & $<0.001$ \\
\hline Expected One-Year BMI Loss $\left(\mathrm{kg} / \mathrm{m}^{2}\right)$ & $11.0 \pm 3.5$ & $9.6 \pm 3.8$ & 6.37 & $<0.001$ \\
\hline \multicolumn{5}{|l|}{ Psychopathology } \\
\hline Binge Eating Scale & $15.0 \pm 8.9$ & $14.8 \pm 9.6$ & 0.41 & 0.681 \\
\hline Symptom Check List 90 (GSI) & $0.8 \pm 0.6$ & $0.8 \pm 0.6$ & -0.82 & 0.412 \\
\hline Body Uneasiness Test (GSI) & $1.8 \pm 1.0$ & $1.5 \pm 1.0$ & 4.26 & $<0.001$ \\
\hline $\begin{array}{l}\text { Three-Factor Eating Questionnaire } \\
\text { Dietary restraint } \\
\text { Disinhibition } \\
\text { Hunger }\end{array}$ & $\begin{array}{l}8.6 \pm 3.9 \\
8.6 \pm 3.5 \\
6.2 \pm 3.6\end{array}$ & $\begin{array}{l}8.5 \pm 3.5 \\
8.5 \pm 3.5 \\
6.4 \pm 3.7\end{array}$ & $\begin{array}{r}-0.59 \\
0.52 \\
-0.95\end{array}$ & $\begin{array}{l}0.553 \\
0.604 \\
0.344\end{array}$ \\
\hline \multicolumn{5}{|l|}{ Primary reason for seeking treatment } \\
\hline Concern for present health, $\mathrm{N}(\%)$ & $146(38.2)$ & $613(53.4)$ & 53.35 & $<0.001$ \\
\hline Concern for future health, $\mathrm{N}(\%)$ & $129(33.8)$ & $384(33.4)$ & & \\
\hline Concern for appearance, $\mathrm{N}(\%)$ & $102(26.7)$ & $137(11.9)$ & & \\
\hline
\end{tabular}


The study sample includes 1530 patients (1194 females and 336 males) enrolled by the 18 centers which collected the 12-month follow-up data; 382 patients (25\%) were classified as young adults, and $1148(75 \%)$ as adults. The two groups showed no significant differences in baseline and maximum BMI, maximum percentage of weight loss and psychopathological characteristics. However, compared with adults, young adults had significantly higher BMI at age 20, higher expected one-year BMI loss and BUT-GSI score, and significantly lower dream and maximum acceptable BMI (Table 1). Moreover, a significantly higher percentage of young adults than adults reported improving appearance as the primary reason for seeking treatment (Table 1).

\section{Treatment outcome}

Among the 500 patients in continuous treatment at 12 months, $101(20.2 \%)$ were young adults, and 399 (79.8\%) adults. Attrition rate was significantly higher in young adults than in adults $(73.6 \%$ vs $65.2 \%$ respectively, ChiSquare $=9.01, p=0.003$ ). The mean percentage of weight loss after 12 months of treatment was significantly higher in young adults $(10.9 \%, \mathrm{SD}=9.8)$ than in adults $(7.2 \%, \mathrm{SD}=7.7, \mathrm{t}=3.46 ; \mathrm{p}=0.001)$. A higher percentage of young adults than adults achieved a weight loss greater than $5 \%(74.5 \%$ vs $60.9 \%$; Chi-Square $=6.22, \mathrm{p}=0.013)$ and $10 \%$ (53.1\% vs 30.8\%; Chi-Square=16.91, p<0.001).

Repeated-measures ANOVA indicated that BMI and the scores of all psychological variables (BUT-GSI, EI scales, SCL-GSI, BES) were significantly reduced in both groups from baseline to 12-month follow-up (Table 2). A significant time $\times$ group interaction was found for
BMI, BES, BUT-GSI and the EI Disinhibition scale, indicating that young adults had a larger BMI reduction and a bigger improvement in these psychosocial variables, compared to adults. Young adults maintained significantly lower SCL-GSI scores than adults both at baseline and after 12 months, as expressed by the significant main effect of group on SCL-GSI.

Repeated-measure ANOVA, using the intention-to-treat approach on the 1530 participants, revealed a significant weight loss from baseline to 12-month follow-up in both groups ( $\mathrm{F}=321.2$, $\mathrm{p}<0.001$ ) but, unlike the completers, no significant difference emerged between young adults and adults on BMI change (Time $\times$ Group: $\mathrm{F}=0.90, \mathrm{p}=0.344$ ).

\section{DISCUSSION}

The main findings of the study are that obese young adults lose more weight and more significantly improve their psychological distress, but have a higher attrition rate than obese adults after 12-month of continuous care.

Compared with subjects studied in a recent behavioral weight loss trials (14), a markedly higher percentage of obese patients seekingtreatment at our medical centers were classified as young adults (25\% vs $7 \%$ ), although in a narrower age-range (25 to 35 years vs 18 to 35 ). This suggests that the scarce rate of enrollment of young adults in weight loss treatment might be a specific problem of research trials, not of real world weight loss treatments.

At baseline, no significant differences in BMI and gender distribution were demonstrated between young adults and adults, but young

\section{TABLE 2}

Psychosocial measures at baseline and at 12 month follow-up in young adults and adults. Data are presented as mean $\pm S D$.

\begin{tabular}{|c|c|c|c|c|c|c|c|}
\hline & \multicolumn{2}{|c|}{$\begin{array}{l}\text { Young adults } \\
\qquad(\mathrm{N}=101)\end{array}$} & \multicolumn{2}{|c|}{$\begin{array}{c}\text { Adults } \\
(\mathrm{N}=399)\end{array}$} & \multicolumn{3}{|c|}{$\begin{array}{l}\text { ANOVA for repeated measures } F \\
\qquad(\mathrm{df}=1,498)\end{array}$} \\
\hline & Baseline & $\begin{array}{l}12 \text { month } \\
\text { follow-up }\end{array}$ & Baseline & $\begin{array}{l}12 \text { month } \\
\text { follow-up }\end{array}$ & Time & Group & TimeXGroup \\
\hline Body Mass Index $\left(\mathrm{kg} / \mathrm{m}^{2}\right)$ & $37.3 \pm 7.6$ & $33.2 \pm 7.7$ & $37.3 \pm 5.4$ & $34.6 \pm 5.4$ & $335.6^{*}$ & 1.2 & $12.7^{*}$ \\
\hline Body Uneasiness Test-GSI & $1.7 \pm 1.0$ & $1.2 \pm 0.9$ & $1.4 \pm 1.0$ & $1.2 \pm 1.0$ & $50.6^{*}$ & 1.5 & $7.7 \S$ \\
\hline Symptom CheckList-90-GSI & $0.7 \pm 0.6$ & $0.5 \pm 0.5$ & $0.8 \pm 0.6$ & $0.7 \pm 0.6$ & $27.6^{*}$ & $6.1 £$ & 1.7 \\
\hline Binge Eating Scale & $14.3 \pm 9.7$ & $9.3 \pm 7.6$ & $13.8 \pm 9.4$ & $10.9 \pm 8.6$ & $87.7^{*}$ & 0.4 & $5.6 £$ \\
\hline $\begin{array}{l}\text { Eating Inventory } \\
\text { Dietary restraint } \\
\text { Disinhibition } \\
\text { Hunger }\end{array}$ & $\begin{array}{l}8.5 \pm 3.4 \\
8.6 \pm 3.3 \\
6.0 \pm 3.7\end{array}$ & $\begin{array}{l}11.8 \pm 3.9 \\
6.6 \pm 3.4 \\
4.6 \pm 3.6\end{array}$ & $\begin{array}{l}8.8 \pm 4.1 \\
8.1 \pm 3.5 \\
6.1 \pm 3.7\end{array}$ & $\begin{array}{l}11.5 \pm 4.4 \\
7.3 \pm 3.7 \\
5.0 \pm 3.6\end{array}$ & $\begin{array}{l}136.5^{*} \\
71.4^{*} \\
45.9^{*}\end{array}$ & $\begin{array}{l}0.0 \\
0.2 \\
0.6\end{array}$ & $\begin{array}{c}1.4 \\
12.4^{*} \\
0.5\end{array}$ \\
\hline
\end{tabular}


adults reported significantly higher body dissatisfaction, larger weight loss expectations and lower dream and maximum acceptable BMIs. Moreover, about $27 \%$ of obese young adults but only about $12 \%$ of obese adults selected "improving appearance" as primary motivation for weight loss. A post-hoc analysis of the adult group, carried out by sub-grouping patients into two nearly equal samples in the age-range $36-50$ and 51-65 $(\mathrm{N}=601$ and $\mathrm{N}=547$, respectively), showed that the age-dependence of these clinical and psychological characteristics extends to the whole spectrum of obese subjects (not reported in details). Aging is associated with a progressive decline in body image dissatisfaction, lower weight loss expectations and lower percentage of appearance as primary motivation for weight loss also in clinical centers principally focused in the clinical management of obesity.

At 12-month follow-up, young adults had significantly higher weight loss than adults (approximately 11\% and 7\%, respectively), but higher attrition rate. Over $70 \%$ of our young adults achieved a weight loss greater than $5 \%$, and over $50 \%$ a weight loss greater than $10 \%$. However, when weight loss was re-analyzed using the intention-to-treat approach, the differences between young adults and adults were no longer significant. This indicates that young adults, when compliant with treatment, reach more satisfying results than adults, but the success is jeopardized by the higher attrition rate among young adults. In contrast with our data, a behavioral weight loss trial showed that young adults had poorer weight loss than adults (14), but differences were no longer significant in completers (14). Future research should investigate differences in recruitment methods and study design, setting of treatment, duration of follow-up, and in patients' environment in order to explain these conflicting results.

Young adults showed a higher reduction in body dissatisfaction, binge eating and disinhibition, than adults. Data are consistent with other studies indicating that the effects on these clinical characteristics are significantly associated with higher weight loss (25), and that younger patients tend to have more psychological benefits from weight loss than older patients (26).

The study has two main strengths. Firstly, it evaluated weight loss and psychological changes in a real world clinical setting. Secondly, it included a large sample of obese young adults, a group rarely considered in obesity treatment research. The study has limitations too. Firstly, we only evaluated young obese subjects seeking treatment in medical settings and, therefore, no inference can be made on the large number of obese young adults who do not seek treatment or who seek help in non-medical (surgical) settings. It is possible that young adults treated in non-medical centers might have different weight loss outcome and attrition. Secondly, the study design did not consider the potential effect of other possible factors (e.g., quality of the weight-loss treatment) on treatment outcome. Thirdly, the high attrition rate limits the external validity of our finding and underlines the difficulties of any long-term evaluation of outcomes in the area of obesity management in the real world. Finally, the study took place in Italy and this limits the external validity of the results to other countries with different health system.

Our results have clinical implications. The high attrition rate of young adults seeking weight loss treatment and the dependence of results on continuous care make strategies to prevent attrition mandatory and a clinical priority in these patients. In young adults on continuous treatment, the probability to attain a healthy weight loss is increased, thus limiting several risk factors for medical complications associated with obesity later in life (27), and improving psychological functioning. Higher weight loss expectations are independent predictors of attrition from weight loss treatment (28); thus addressing unrealistic expectations of obese young adults might be a potential strategy to reduce their rate of attrition. Future studies should investigate this important issue.

\section{ACKNOWLEDGEMENTS}

The QUOVADIS Study was supported by an unrestricted grant from Bracco SpA, Milan (Italy). A complete list of participants in the QUOVADIS study was previously published (Diab Nutr Metab 2003; 16: 115-24).

\section{REFERENCES}

1. Ogden CL, Carroll MD, Curtin LR, et al. Prevalence of overweight and obesity in the united states, 1999-2004. JAMA 2006; 295: 1549-55.

2. Pagano R, La Vecchia C, Decarli A, et al. Trends in overweight and obesity among italian adults, 1983 through 1994. Am J Public Health 1997; 87: 1869-70.

3. Truesdale KP, Stevens J, Lewis CE, et al. Changes in risk factors for cardiovascular disease by baseline weight status in young adults who maintain or gain weight over 15 years: The cardia study. Int J Obes (Lond) 2006; 30: 1397-407.

4 Lewis CE, Jacobs DR jr, McCreath H, et al. Weight gain continues in the 1990s: 10-year trends in weight and overweight from the cardia study. Coronary artery risk development in young adults. Am J Epidemiol 2000; 151: 1172-81. 
5. The NS, Suchindran C, North KE, et al. Association of adolescent obesity with risk of severe obesity in adulthood. JAMA 2010; 304: 2042-7.

6. Norman JE, Bild D, Lewis CE, et al. The impact of weight change on cardiovascular disease risk factors in young black and white adults: The cardia study. Int J Obes Relat Metab Disord 2003; 27: 369-76.

7. Brennan SL, Cicuttini FM, Pasco JA, et al. Does an increase in body mass index over 10 years affect knee structure in a population-based cohort study of adult women? Arthritis Res Ther 2010; 12: R139.

8. Levine MD, Klem ML, Kalarchian MA, et al. Weight gain prevention among women. Obesity (Silver Spring) 2007; 15: 1267-77.

9. Eiben G, Lissner L. Health hunters - an intervention to prevent overweight and obesity in young high-risk women. Int J Obes (Lond) 2006; 30: 691-6.

10. Hivert MF, Langlois MF, Berard P, et al. Prevention of weight gain in young adults through a seminarbased intervention program. Int J Obes (Lond) 2007; 31: 1262-9.

11. Stice E, Shaw H, Marti CN. A meta-analytic review of obesity prevention programs for children and adolescents: The skinny on interventions that work. Psychol Bull 2006; 132: 667-91.

12. Jeffery RW, French SA. Preventing weight gain in adults: The pound of prevention study. Am J Public Health 1999; 89: 747-51.

13. Poobalan AS, Aucott LS, Precious E, et al. Weight loss interventions in young people (18 to 25 year olds): A systematic review. Obes Rev 2010; 11: 580-92.

14. Gokee-LaRose J, Gorin AA, Raynor HA, et al. Are standard behavioral weight loss programs effective for young adults? Int J Obes (Lond) 2009; 33: 1374-80.

15. Melchionda N, Marchesini G, Apolone G, et al. The QUOVADIS study. Features of obese italian patients seeking treatment at specialist centers. Diabetes Nutr Metab 2003; 16: 115-24.

16. Erikson EH. Childhood and society. New York, WW Norton \& Co, Inc, 1950.

17. Dalle Grave R, Calugi S, Magri F, et al. Weight loss expectations in obese patients seeking treatment at medical centers. Obes Res 2004; 12: 2005-12.

18. Derogatis LR, Cleary PA. Confirmation of the dimensional structure of the scl-90: A study in construct validity. J Clin Psychol 1977; 33: 981-9.

19. Stunkard AJ, Messick S. The three-factor eating questionnaire to measure dietary restraint, disinhibition and hunger. J Psychosom Res 1985; 29: 71-83.

20. Stunkard AJ, Messick S. The eating inventory. San Antonio, TX, Psychological Corporation, 1988.

21 Gorman BS, Allison DB. Measures of restrained eating. In Allison DB (Ed) Handbook of assessment for eating behaviors and weight-related problems. Thousands Oaks, Ca, Sage Publishing, Inc, 1995, pp 149-84.

22. Cuzzolaro M, Vetrone G, Marano G, et al. The body uneasiness test (BUT): Development and validation of a new body image assessment scale. Eating Weight Disord 2006; 11: 1-13.

23. Marano G, Cuzzolaro M, Vetrone $G$, et al; and the QUOVADIS Study Group. Further validation study of the body uneasiness test (BUT) in a clinical sample of 1922 adult obese subjects. Eating Weight Disord 2007; 12: $70-82$.

24. Gormally J, Block S, Daston S, et al. The assessment of binge eating severity among obese persons. Addict Behav 1982; 7: 47-55.

25. Hainer V, Kunesova M, Bellisle F, et al. Psychobehavioral and nutritional predictors of weight loss in obese women treated with sibutramine. Int J Obes (Lond) 2005; 29: $208-16$

26. Dalle Grave $R$, Cuzzolaro M, Calugi $S$, et al. The effect of obesity management on body image in patients seeking treatment at medical centers. Obesity (Silver Spring) 2007; 15: 2320-7.

27. National Institutes of Health: Clinical guidelines on the identification, evaluation, and treatment of overweight and obesity in adults - the evidence report. Obes Res 1998; 6 (Suppl 2): 51S-209S.

28. Dalle Grave R, Calugi S, Molinari E, et al. Weight loss expectations in obese patients and treatment attrition: An observational multicenter study. Obes Res 2005; 13 : 1961-9. 